\title{
Der Kampf um transnationale Gerechtigkeit. Neue Chancen für die Menschenrechtsbewegung?1
}

\section{Transnationale Bewegungen und Gegenbewegungen}

Die Menschenrechtsbewegung hat ihr Repertoire und ihre Aktionsspielräume maßgeblich erweitert. Ob man diese Entwicklung »Globalisierung von unten« oder »Transnationalisierung des Rechts « nennt oder ob man nur das Eingreifen weiterer transnationaler Akteure konstatiert, das Phänomen erscheint bereits vertraut. Es ist aber relativ jung, auf wenige Staaten und Themen beschränkt und für alle staatlichen sowie nicht-staatlichen Beteiligten in der Praxis noch ungewohnt. Einige Beispiele:

Das Joint Venture zwischen der US-amerikanischen Ölfirma »Unocal Cooperation«, der französischen »Total S.A.« und der burmesischen »Myanmar Oil and Gas Enterprise« ließ I995 die Yadana-Pipeline mit Hilfe der burmesischen Armee durch Sklavenarbeit unter Folterungen, Vergewaltigungen und Tötungen der ArbeiterInnen bauen. Tausende DorfbewohnerInnen der Umgebung wurden vertrieben. Die Opfer klagten mit Hilfe des US-amerikanischen Center for Constitutional Rights (CCR) in den USA auf Schadensersatz nach dem Alien Torts Claims Act (ATCA). ${ }^{2}$ Die französische Organisation Sherpa strengte ein Strafverfahren in Belgien sowie eine zivile Schadensersatzklage in Frankreich an. ${ }^{3}$ Sowohl Unocal als auch Total entschädigten nach mehrjährigen Schadensersatzprozessen die Opfer in unbekannt gebliebener Höhe.

Am 3 I.I 2.2003 wird der gebürtige Libanese und deutsche Staatsbürger Khaled El Masri an der serbisch-mazedonischen Grenze festgenommen und später von US-amerikanischen Staatsbürgern über Bagdad nach Afghanistan verbracht, wo er misshandelt und gefoltert wird. Am 28.5.2004 wird er über Albanien nach Deutschland ausgeflogen. Dort werden Ermittlungen aufgenommen, aufgrund derer das Amtsgericht München im Januar 2007 gegen I3 mutmaßliche CIAAgenten und Flugpersonal einen Haftbefehl erlässt. Im Juni 2008 klagt das »European Center for Constitutional and Human Rights (ECCHR)« beim Verwaltungsgericht Berlin mit einer Leistungsklage gegen die Bundesrepublik Deutschland mit dem Ziel, dass die BRD die Auslieferung der mit Haftbefehl des Amtsgericht München gesuchten US-Staatsbürger beantragt. Die »American Civil Liberties Union (ACLU) « hatte bereits im Dezember 2005 eine Schadensersatzklage in den USA u.a. gegen den ehemaligen CIA-Direktor George Tenet eingelegt. In Albanien und Mazedonien erstrebt die »Open Society Justice Initiative « auf der Grundlage von Informationsfreiheitsgesetzen die Freigabe von dortigen Regierungsinformationen und legt wegen der Geschehnisse in Mazedonien Strafanzeige ein. Weiterhin wurde der Fall zunächst in Mallorca und später vor dem Ermittlungsrichter der Audiencia Nacional in Madrid als

\footnotetext{
I Für ihre Anmerkungen danke ich Miriam Saage-Maaß.

2 US Court for Appeals for the $9^{\text {th }}$ Circuit, 2002 US App LEXIS 19263 ( th $^{\text {th }}$ Cir 2002), vgl. auch http:// www.ccrjustice.org/newsroom/press-releases/historic-advance-universal-human-rights\% $3 \mathrm{~A}$-unocalcompensate-burmese-villagers http://www.philodroit.be/IMG/pdf/B._FRYDMAN_-_

3 Court des Cassation de Belgique, P.04.0482. F/I und P.07.003 I.F/I sowie Tribunal de Grande Instance, Nanterre; vgl. www.asso-sherpa.org.
} 
flüge, untersucht. ${ }^{4}$

Zwei Beispiele von Völkerrechtswidrigkeiten, deren Ahndung durch strafrechtliche und zivilrechtliche Verfahren angestrebt wird. Den Joint Ventures französischer und US-amerikanischer Ölkonzerne mit burmesischen Armeeangehörigen und der Kooperation der CIA mit mazedonischen, afghanischen und albanischen Sicherheitskräften folgt die Gegenbewegung US-amerikanischer, französischer, belgischer, deutscher, spanischer, mazedonischer und albanischer JuristInnen und Menschenrechtsorganisationen. Es geht um Sachverhalte, die sich entweder in verschiedenen Nationalstaaten zutragen oder über das nationalstaatliche Territorium hinaus wirken. Auf der Verletzer- und auf der Verletztenseite sind oftmals Akteure verschiedener Nationalitäten beteiligt. Auf alle geschilderten Sachverhalte findet hybrides Recht, also das Recht unterschiedlicher nationaler Rechtsordnungen sowie internationales Recht, Anwendung. Vor allem aber zeichnen sich die Sachverhalte dadurch aus, dass vornehmlich nichtstaatliche Akteure aus der Menschenrechtsbewegung die verschiedenen juristischen Verfahren initiiert haben, sie betreiben und begleiten. So wäre wohl keines der oben knapp geschilderten zehn juristischen Verfahren ohne die maßgebliche Beteiligung zivilgesellschaftlicher Akteure in Gang gekommen.

\section{Der Pinochet- und Videla-Effekt: Die europäischen und deutschen Straf- verfabren gegen argentinische Militärs (1995-2008)}

Das Völkerstrafrecht ist das Rechtsgebiet, in dem nicht-staatliche Akteure, transnational vernetzt, in auffälliger Weise sowohl bei der Herausbildung des Rechts mitwirken und neuartige Formen der Rechtsanwendung in und jenseits der primär zuständigen Nationalstaaten nutzen, wie das Beispiel des seit I995 anhängigen Komplexes der europäischen und deutschen Strafverfahren gegen argentinische Militärs zeigt.

Die Verfolgung von Völkerstraftaten ist klassischerweise Sache der Staaten gewesen. Bei den Alliierten Militärtribunalen nach dem Zweiten Weltkrieg spielten zivilgesellschaftliche Organisationen ebenso wenig eine Rolle wie bei den wenigen NS-Verfahren, die im Täterland Deutschland stattfanden. Eine wie auch immer geartete Beteiligung an den wenigen Völkerstrafverfahren nach dem Zweiten Weltkrieg gehörte nicht zum Repertoire von Menschenrechtsorganisationen. Als eine der wenigen Ausnahmen ist die Antikriegsbewegung in den USA anzusehen, die Kriegsverbrechen der US-Army im laufenden Vietnamkrieg aufzuklären und Armeeangehörige, wenn auch nur auf symbolischen Meinungstribunalen wie dem Russell-Tribunal,' anzuklagen versuchte. Die USMenschenrechtsbewegung spielte dann eine Pionierrolle zwar nicht im Völkerstrafrecht, aber im Zivilrecht, ${ }^{6}$ als nämlich I 980 das »Center for Constitutional Rights « in New York mit Hilfe der bis dahin bedeutungslosen Vorschrift aus dem Jahre I789, dem Alien Torts Claims Act (ATCA), eine Verurteilung des, inzwischen in den USA lebenden, paraguayanischen Polizisten Pena-Irala wegen der 1976 in Paraguay erfolgten Folterung und Ermordung des paraguayinischen

\footnotetext{
4 Vgl. zu allen Verfahren m.w.N.: European Center for Constitutional and Human Rights (Hrsg.), CIA"Extraordinary Rendition«-Flights, Torture And Accountability, A European Approach, März 2008.

5 Instruktiv hierzu Francois Rigaux, Internationale Tribunale nach den Nürnberger Prozessen, in: Hankel/ Stuby (Hrsg.), Strafgerichte gegen Menschheitsverbrechen, Hamburg 1995, I 42 ff.

6 Das US-amerikanische Zivilrecht trägt allerdings strafrechtliche Elemente, u.a. punitive damages, in sich.
} 

Dollar erstritt.7

Die weltweit vernetzten Menschenrechtsorganisationen - der »Coalition for the International Criminal Court $\ll^{8}$ gehören über 2.500 NGOs an - waren dann bei der Ausarbeitung des Rom-Statutes für einen Internationalen Strafgerichtshof I 998 und der Errichtung desselben im Jahre 2002 aktiv. Insbesondere spielt die Menschenrechtsbewegung bei der Aufklärung und Strafverfolgung von Menschenrechtsverletzungen vor nationalen Gerichten, sei es in den Tatortstaaten oder in dritten Staaten, eine große Rolle. So gehen die chilenische und argentinische Menschenrechtsbewegung seit den I 970er Jahren mit juristischen Mitteln gegen die Straflosigkeit der Diktaturverbrechen in ihren Ländern vor. In Argentinien waren bereits 1985, unmittelbar nach Ende der Diktatur, fünf der neun angeklagten Kommandeure wegen Mordes, Freiheitsberaubung, Folter, Nötigung oder Raub zu langjährigen, teilweise auch lebenslangen Freiheitsstrafen verurteilt worden. Aufgrund des enormen politischen und militärischen Drucks der Militärs nach den ersten Verurteilungen erließ die demokratische Regierung eine Serie von Amnestiegesetzen bzw. Gnadenakten gegen verurteilte Militärs. Trotz dieser Gesetze kämpften die argentinischen Menschenrechtsorganisationen um die Aufklärung der Verbrechen und die Strafverfolgung der Militärs, zunächst in Argentinien und Lateinamerika und ab Mitte der I990er Jahre in Europa. ${ }^{9}$ Die Initialzündung erfolgte in Spanien, als von MenschenrechtlerInnen I995 eingelegte Strafanzeigen gegen argentinische und später gegen chilenische Militärs nicht nur zu der spektakulären Verhaftung des chilenischen Ex-Diktators Augusto Pinochet in London 1998 führten. Die Anzeigen lösten umfangreiche Strafverfolgungsaktivitäten der spanischen Justiz aus, und der argentinische Ex-Kapitän der Marine, Adolfo Scilingo, wurde 2005 von der Audiencia Nacional u.a. wegen 30 Tötungen zu einer Gesamtstrafe von 63 Jahren verurteilt.

Die Bedeutung der spanischen Verfahren liegt zum einen darin, dass die spanische Justiz mit einem hohen Aufwand in den beiden historischen Komplexen Chile und Argentinien ermittelte, wobei sich die Ermittlungen über die untersuchten Einzelfälle hinaus auch auf die Rolle der USA und die Geheimdienstzusammenarbeit im Rahmen der »Operation Condor ${ }^{10}$ erstreckten.

Die spanische Justiz betreibt bis heute europaweit in einmaliger Weise die Strafverfolgung von Menschenrechtsverbrechen nach dem Weltrechtsprinzip in einem Dutzend Fällen. Ihr Wirken war folgenreich für die Ausbreitung des Weltrechtsprinzips im Allgemeinen, hatte einen großen Einfluss auf die (Wieder-)Aufnahme der Strafverfolgung in den primär zuständigen Tatortstaaten Chile und Argentinien und diente als Modell für weitere Strafverfolgung in anderen europäischen Staaten wie Italien und Frankreich.

7 Filartiga v. Pena Irala, 630 F 2d 876 ( $2^{\text {nd }}$ Cir. 1980); vgl. dazu u.a. Claudia Hailer, Menschenrechte vor Zivilgerichten. Die Human Rights Litigation in den USA, Berlin 2006.

8 Http://www.iccnow.org.

9 Vgl. Florian Jessberger, Von der Pflicht des Staates, Menschenrechtsverletzungen zu untersuchen, Kritische Justiz I 996, 290 ff.; ders., Prosecuting International Crimes in Domestic Courts, Finnish Yearbook for International Law 200I, 28 I ff.

Io Im Jahre 1975 vereinbarten die Diktaturen in Brasilien, Uruguay, Paraguay, Chile, Peru, Bolivien und in Argentinien eine sehr weitgehende Zusammenarbeit mit den USA im Rahmen der sogenannten »Operación Condor«. Informationen über Regimegegner wurden von den Geheimdiensten erfasst und ausgetauscht und diese über die Landesgrenzen verfolgt, ausgeliefert und teilweise liquidiert, u.a. General Prats in Argentinien und der chilenische Ex-Außenminister Orlando Letellier in Washington. 
In Deutschland betreibt das Menschenrechtsnetzwerk » Koalition gegen Straflosigkeit « seit 1998 nach dem spanischen Beispiel Strafverfahren im Namen von 39 Opfern gegen zeitweilig 89 argentinische Militärs. ${ }^{\text {I }}$ Nach umfangreichen Ermittlungen der Staatsanwaltschaft Nürnberg-Fürth wurden 2003 Haftbefehle des Amtsgerichts Nürnberg wegen mittelbarer Täterschaft gegen die ehemaligen Staats- und Militärjunta-Chefs Jorge Rafael Videla und Emilio Eduardo Massera wegen der Morde an den Deutschen Elisabeth Käsemann und Klaus Zieschank erlassen, aufgrund derer die Bundesregierung Auslieferungsverfahren initiiert hat.

Die Koalition untersuchte neben den Verbrechen der argentinischen Militärdiktatur auch die Rolle der deutschen Diplomatie, Politik und Wirtschaft und deren intensive Beziehungen zur Militärdiktatur. ${ }^{\mathrm{I}}{ }^{2}$ Beispielhaft für die Verwicklung transnationaler Konzerne in die Menschenrechtsverletzungen der argentinischen Militärdiktatur ist der bei den Staatsanwaltschaften Nürnberg und Buenos Aires und US-Zivilgerichten anhängig gemachte Fall der I4 verschwundenen GewerkschafterInnen bei Mercedes Benz Argentinien, die 1976/77 unter Mithilfe der Firma verschleppt und später getötet wurden. ${ }^{\mathrm{I}} 3$

Die Aktivitäten anlässlich der Pinochet-Verhaftung, aber auch der Erlass von Haftbefehlen sowie die Verurteilungen in Deutschland und anderen europäischen Ländern, wurden in der Öffentlichkeit als großer Erfolg bewertet. Die europäischen Verfahren haben den Druck auf die argentinische Justiz massiv erhöht, selbst in die Aufarbeitung der damaligen Verbrechen einzutreten. ${ }^{\mathrm{I}}$ Naomi Roht-Arriaza ${ }^{15}$ sieht im Pinochet-Fall das Resultat der Aktivitäten einer losen Allianz von nationalen und internationalen MenschenrechtsaktivistInnen, Familienangehörigen von Opfern, RechtsanwältInnen, JournalistInnen und WissenschaftlerInnen auf der einen und RichterInnen und Regierungen auf der anderen Seite. Dieses Zusammenwirken institutioneller und nicht-institutioneller Akteure habe zunächst zur Verhaftung Pinochets in London 1998 und danach zu dem von ihr so bezeichneten »Pinochet-Effekt « geführt, einer Rückwirkung der internationalen Diskussionen, Bemühungen und Strafverfolgungstätigkeiten auf die Aufnahme ernsthafter Ermittlungen und Strafverfolgung in Chile - und auch in Argentinien. Dort wurden ab 2005, nach der Annullierung der Amnestiegesetze, zahlreiche Ermittlungsverfahren gegen Militärs (wieder) eröffnet, in denen Haftbefehle gegen etwa I 50 Tatverdächtige erlassen und erste Verurteilungen ausgesprochen wurden.

\section{Transnational Justice?}

Die Menschenrechtsbewegung nutzt also, wie die Beispiele zeigen, zunehmend transnational rechtliche Verfahren, um Menschenrechtsverletzungen aufzuklären und ahnden zu lassen. Sie reagiert damit darauf, dass Menschenrechtsverlet-

I I Vgl. www.menschenrechte.org/koalition.

I 2 Vgl. Koalition gegen Straflosigkeit (Hrsg.), Menschenrechte und Außenpolitik, Bad Honnef 2006.

I 3 Das Verfahren ist nach einem den Antrag im Klageerzwingungsverfahren verwerfenden Beschluss des OLG Nürnberg vom 2005 vorerst abgeschlossen. Die in den USA vom International Labour Rights Fund am 2004 eingelegte zivilrechtliche Entschädigungsklage gegen Daimler Chrysler ist derzeit noch anhängig.

I4 Denis Basak, Die dritte Entrechtung. Zum Umgang der Staatsanwaltschaft Nürnberg-Fürth mit Ermittlungsverfahren gegen Angehörige der argentinischen Militärjunta wegen getöteter Nachkommen jüdischer Flüchtlinge aus Nazi-Deutschland, Zeitschrift für Internationales Strafrecht 2007, $374 \mathrm{ff}$.

Is Dies., The Pinochet Effect. Transnational Justice in the Age of Human Rights, Philadelphia 2005. 
zungen in transnationalen Zusammenhängen, wie beispielsweise die Operation Condor in den I970er Jahren in Lateinamerika und aktuell das »Extraordinary Rendition-Programme " der CIA und von transnational agierenden Akteuren, bzw. durch Unternehmen wie Unocal, Shell oder Total, begangen werden.

Wegen der Besonderheiten der amerikanischen Rechtskultur und dank einer pragmatisch ausgerichteten und professionell arbeitenden BürgerInnen- und Menschenrechtsbewegung haben sich Formen der transnationalen juristischen Durchsetzung von Menschenrechten bereits seit 1980 in den Alien Torts Claims Act (ATCA)-Fällen herausgebildet, wenn sie auch dort auf zahlreiche politische und juristische Hindernisse stößt. Diese transnationale Menschenrechtsarbeit ist auch eine Reaktion auf die Globalisierung, die sich in diesem Bereich u.a. in der häufigen Präsenz von Tätern und Opfern in den USA, den weltweiten Aktivitäten der US-Regierung und der in den USA präsenten transnationalen Konzerne äußert. Mittlerweile klagen US-Bürgerrechtsorganisationen aus den USA u.a. gegen die CIA-Entführungsflüge, die Folterungen in Abu Gbraib und Guantánamo. Dabei sind die Erfolge der Guantánamo-AnwältInnen hervorzuheben: Das Center for Constitutional Rights übernahm Anfang 2002 in aussichtsloser Position die Vertretung der Gefangenen, um bis heute mit einem Team von über 500 AnwältInnen, auch aus großen Anwaltsfirmen, drei Mal vor dem USSupreme Court, zuletzt in Sachen Boumediene et.al vs. Bush im Juni 2008, ${ }^{16} \mathrm{zu}$ obsiegen. Ihre Aktivitäten haben sowohl in professioneller Hinsicht (durch Fundraising finanziertes Public Interest Lawyering, begleitende Öffentlichkeitsarbeit) als auch inhaltlich (Gebrauch des Rechts als Instrument im Kampf um gesellschaftlichen Fortschritt, Gerichte als Foren des Protests) weltweit Vorbildcharakter für Menschenrechtsorganisationen, weniger in Europa als in Lateinamerika, West- und Südafrika und Indien.

In Westeuropa etablierten sich eher lokal und thematisch begrenzte Netzwerke, die vor allem im Bereich des Weltrechtsprinzips in vielen west- und nordeuropäischen Staaten Verfahren wegen extraterritorialer Völkerstraftaten initiiert und an diesen mitgewirkt haben. Die Wirkungen dieses transnationalen Rechtsgebrauchs durch die Menschenrechtsbewegung wurden bereits angedeutet:

I. Der Pinochet- und Videla-Effekt: Das an einem Ort begangene Unrecht findet aufgrund der Methoden der Menschenrechtsbewegung an einem anderen Ort Beachtung. Dort wird die institutionelle oder justizielle Aufarbeitung ganz oder teilweise betrieben, um dann (bestenfalls) am ursprünglichen Ort fortgesetzt zu werden.

2. Der kreative Gebrauch bestehender Normen führt zu Innovationen des Menschenrechtsschutzes und damit in gewisser Weise zur Schöpfung neuen Rechts, wie beim ATCA in den USA und beim Prinzip der Universellen Jurisdiktion in Europa zu beobachten ist.

3. Dabei sind Protestbewegungen an der Produktion neuen Rechts maßgeblich beteiligt wie die Mütter des Plaza de Mayo, die die Repressionstechnik des Verschwindenlassens öffentlich skandalisierten und juristisch angriffen. Aufgrund der argentinischen Erfahrungen findet sich dieser Verbrechenstatbestand im Rahmen der Verbrechen gegen die Menschlichkeit in allen neueren Statuten für internationale Gerichtshöfe, im deutschen Völkerstrafgesetzbuch und der UN-Konvention zum Verschwindenlassen. ${ }^{17}$ 
Skeptiker wie David Kenned $y^{18}$ werfen der Menschenrechtsbewegung eine mitunter verengte Sichtweise vor: Ihre Instrumente behandelten bisweilen, selbst wenn sie erfolgreich sind, nur die Symptome und nicht die Krankheit. Selbst bei Kompensation der Opfer würde dieselbe Verteilung von Macht und Reichtum bestehen bleiben, die zu den Menschenrechtsverletzungen führte.

Dieser Kritik ist dann Recht zu geben, wenn die Menschenrechtsbewegung sich auf eine bloß reaktive, rückwärts gewandte und ausschließlich juristische Vorgehensweise gegen Menschenrechtsverletzungen beschränkt. Um solchen negativen Effekten einer transnationalen Menschenrechtsarbeit vorzubeugen, sollte man der argentinischen Menschenrechtsbewegung darin folgen, die politischökonomischen Zusammenhänge, aus denen heraus Menschenrechtsverletzungen begangen werden, ebenso auf die Agenda zu setzen wie die Komplizenschaft von Akteuren aus den Ländern des Nordens - wie zum Beispiel in dem oben geschilderten Fall von Mercedes Benz in Argentinien ${ }^{19}$ - und sich dabei neben juristischen auch künstlerischer, politischer und sozialer Mittel bedienen. Die aktuelle ökonomische Situation und deren Ursachen sollten dabei ebenso thematisiert werden wie die rechtliche Durchsetzung wirtschaftlicher, sozialer und kultureller Rechte, die gleichberechtigt neben der Durchsetzung politischer und bürgerlicher Rechte steht. Der Gefahr des Paternalismus und der Lenkung der Menschenrechtsarbeit durch finanzstarke westliche staatliche und nicht-staatliche Agenturen kann dadurch entgegengesteuert werden, dass transparente Strukturen geschaffen werden und eine stetige öffentliche Diskussion stattfindet. Derartig kontextualisiert kann transnationale juristische Menschenrechtsarbeit nicht nur zur Verbesserung des Menschenrechtsschutzes, sondern - in dem von Kennedy geforderten pragmatischen Sinn - zur tatsächlichen Verbesserung von gesellschaftlichen Missständen beitragen.

I7 Andreas Fischer-Lescano, Globalverfassung. Die Geltungsbegründung der Menschenrechte, Weilerswist 2005 .

I 8 David Kennedy, The International Human Rights Movement: Part of the Problem?, Harvard Human Rights Journal 2002, I0I ff.; ders., The Darker Sides of Virtue: Reassessing International Humanitarianism, 2005.

I9 Siehe dazu auch Naomi Klein, Die Schock-Strategie, Frankfurt am Main 2007, I 54 ff. 